\title{
L'enseignement de l'évolution dans les manuels scolaires de huit pays riverains de la Méditerranée
}

Marie-Pierre Quessada, Pierre Clément, Sabah Selmaoui et Adriana Valente

\section{(2) OpenEdition}

\section{Journals}

Édition électronique

URL : http://journals.openedition.org/trema/2651

DOI : $10.4000 /$ trema. 2651

ISSN : 2107-0997

Éditeur

Faculté d'Éducation de l'université de Montpellier

Édition imprimée

Date de publication : 1 décembre 2011

Pagination : $21-35$

ISSN : 1167-315X

\section{Référence électronique}

Marie-Pierre Quessada, Pierre Clément, Sabah Selmaoui et Adriana Valente, «L'enseignement de l'évolution dans les manuels scolaires de huit pays riverains de la Méditerranée », Tréma [En ligne], 35 36 | 2011, mis en ligne le 01 décembre 2013, consulté le 30 avril 2019. URL : http:// journals.openedition.org/trema/2651 ; DOI : 10.4000/trema.2651

Ce document a été généré automatiquement le 30 avril 2019.

Trema 


\title{
L'enseignement de l'évolution dans les manuels scolaires de huit pays riverains de la Méditerranée
}

\author{
Marie-Pierre Quessada, Pierre Clément, Sabah Selmaoui et Adriana \\ Valente
}

\section{Introduction}

«Rien n'a de sens en biologie si ce n'est à la lumière de l'évolution» (DOBZANSKY, 1973). Depuis DARWIN, l'ensemble des recherches en sciences de la Vie et de la Terre a acquis une grande cohérence grâce à la théorie de l'évolution, c'est un modèle explicatif et heuristique de l'histoire de la vie qui traverse tous les champs de la biologie et de la géologie. Cette théorie fait consensus pour l'ensemble de la communauté scientifique qui la soumet à rectification par de nouveaux questionnements, de nouvelles données factuelles. Ainsi l'origine des espèces vivantes par des variations suivies de sélection naturelle proposée par DARWIN est admise par les biologistes, même si d'autres processus évolutifs ont depuis été mis en évidence pour expliquer comment de nouvelles espèces peuvent émerger à partir de modifications génétiques au sein de populations d'espèces préexistantes (dérive génétique, flux de gènes entre espèces, etc.).

En ce début de XXIe siècle, les enseignements sur l'évolution sont pourtant fortement exposés aux attaques anti-évolutionnistes, ce qui suscite des réactions du milieu scientifique dont la déclaration solennelle de l'Inter academy panel (IAP, 2006) représentant les Académies des sciences de 68 pays. Les enjeux des recherches en didactique sur ce thème sont grands. Il s'agit d'exercer une grande vigilance épistémologique sur les contenus de l'enseignement scientifique. En effet, dans ce domaine de l'origine des êtres vivants actuels, en particulier celle de l'espèce humaine, les interprétations scientifiques sont souvent en interaction avec des croyances ou des opinions. Comme le dit Pascal PICQ (2002), «le véritable problème de la paléoanthropologie, c'est une certaine idée de l'homme». Les enseignants, comme les 
chercheurs, ont à se méfier de leurs propres conceptions influencées notamment par l'anthropocentrisme et le finalisme. L'anthropocentrisme est une conception qui considère l'homme comme le centre du monde, elle correspond à la place qu'occupe l'espèce humaine au sein du monde vivant dans la culture judéo-chrétienne. Le finalisme ou «théorie des causes finales» est une conception selon laquelle toute évolution tend vers la réalisation d'un but fixé d'avance, par exemple l'émergence de l'espèce humaine. Il fait souvent intervenir une (ou des) force(s) transcendantale(s) à la matière et à la vie, ce qui s'oppose à une approche scientifique rationnelle. Il est donc nécessaire de clarifier l'approche scientifique, en distinguant le message scientifique des valeurs, opinions et idéologies qui y sont associées. Le présent travail propose une analyse comparée des manuels scolaires de biologie de huit pays méditerranéens. Nous tenterons de mettre en lien les convergences et les divergences dans le traitement de l'évolution dans ces manuels avec les éléments culturels, géographiques, linguistiques, économiques et sociaux qui lient et différencient ces huit pays méditerranéens.

\section{Cadre théorique et problématique}

3 Si de nombreux travaux de recherche sur les manuels scolaires portent sur l'analyse de leurs contenus avec une centration sur les aspects idéologiques et culturels (cHoppin, 1992), peu s'inscrivent dans le domaine des sciences. En ce qui concerne le thème de l'évolution, матну (1997) a montré la présence d'idéologies implicites dans les manuels belges étudiés. TIDON et LEWONTIN (2004) font un bilan de plusieurs recherches concernant le matériel pédagogique et les programmes relatifs à l'enseignement de l'évolution dans le monde. Une étude des manuels de biologie des High Schools des USA au XXe siècle indique une relation entre le développement donné à l'évolution humaine dans ces manuels et les influences sociales du moment (sKOOG, 2005). Notre recherche a d'abord porté sur l'évolution des programmes et manuels scolaires français au cours des XIXe et XXe siècles (QUESSADA et CLÉMENT, 2007), montrant que le délai de transposition didactique des découvertes sur les origines de l'espèce humaine variait en fonction du contexte socioculturel de l'époque. Nous avons ensuite élargi l'étude à 19 pays et montré que des implicites idéologiques s'exprimaient largement dans les arbres d'évolution (schémas qui récapitulent les filiations entre les espèces à partir d'un ancêtre commun) des manuels de ces différents pays (QUESSADA et al., 2008).

4 Notre recherche se situe à l'intersection de plusieurs approches: didactique, épistémologique et socioculturelle. L'approche historique et épistémologique a conduit à identifier des obstacles épistémologiques à l'avancée des connaissances scientifiques et à catégoriser les conceptions relatives à l'évolution et en particulier l'évolution humaine ( QUESSADA, 2008). Sur le plan didactique, nous nous intéressons aux manuels scolaires dans le contexte théorique de la transposition didactique. La modélisation de la transposition didactique et de l'analyse des conceptions des enseignants propose trois grands types de référence en interaction, selon le modèle KVP : les connaissances scientifiques (K pour Knowledge), les valeurs (V) et les pratiques sociales (P) (CLÉMENT, 2004). Le présent travail s'intéresse aux interactions entre science et valeurs, lors de la transposition didactique du concept d'évolution biologique dans les manuels scolaires de huit pays méditerranéens. Ces résultats seront ensuite discutés en fonction des conceptions des enseignants dans ces mêmes pays, conceptions analysées dans des travaux précédents (CLÉMENT et QUESSADA, 2008, 2009). 


\section{Méthodologie}

Ce travail a été développé dans le cadre du projet européen Biohead-Citizen, «Biology, health and environmental education for better Citizenship ${ }^{1} »$. Vingt équipes de recherche de dix-neuf pays, ont été associées à ce projet mené de 2004 à 2008. Le travail présenté ici est centré sur les huit pays méditerranéens parties prenantes du projet. Quatre pays sont européens - Chypre, France, Italie et Malte - et quatre pays sont d'Afrique du Nord et du Moyen Orient: Algérie, Liban, Maroc et Tunisie. Ils ont été choisis pour permettre des études comparatives dans des contextes géographiques, sociaux, économiques, historiques différents.

\section{1. Les programmes scolaires}

6 Une étude préliminaire portant sur les programmes scolaires de 2004 a été effectuée dans chaque pays. Nous avons établi un tableau comparatif des programmes des différents pays de la première classe de scolarité obligatoire à la dernière classe avant l'université. Cette première approche des programmes scolaires avait pour but de guider le choix des manuels scolaires à collecter et étudier dans chaque pays.

\section{2. Les manuels scolaires}

7 Le corpus est constitué de 18 manuels (tableau 1) provenant des huit pays méditerranéens. Le corpus de chaque pays a été constitué par l'équipe de recherche nationale impliquée dans le projet Biohead sur la base des manuels scolaires en vigueur dans les classes en 2004. C'est le cas de 17 manuels, 16 présentant une partie traitant de l'évolution ( 3 chypriotes, 4 français, 5 italiens, 1 libanais, 2 maltais, 1 tunisien), 1 traite de la préhistoire (1 algérien). Nous avons ajouté pour cette publication un manuel datant de 2007 quand l'évolution a été réintroduite au Maroc (1 marocain). Dans la plupart des pays, il n'existe qu'une édition nationale, auquel cas, il n'y a pas de choix d'éditeurs à effectuer. Pour l'Italie et la France, le choix des équipes s'est porté vers les maisons d'édition ayant la plus grande part de marché. L'étude s'est focalisée sur les manuels des classes terminales, niveau d'enseignement de l'évolution dans les pays dont les programmes ne traitent ce thème qu'à un seul niveau. 


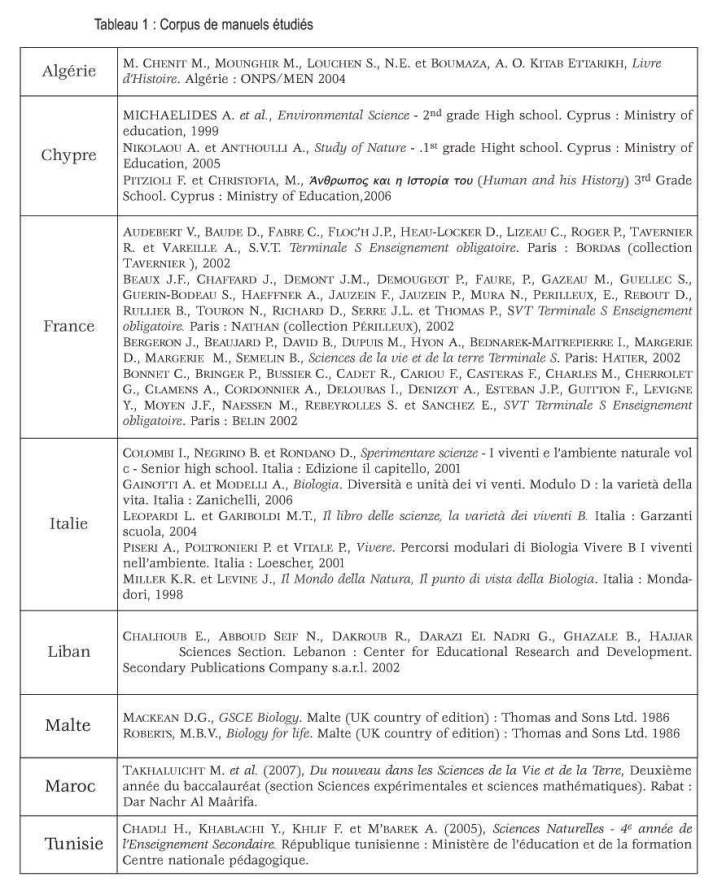

Nous avons élaboré une grille d'analyse des manuels à partir d'une étude préalable ayant identifié les obstacles épistémologiques relatifs à l'évolution (QUESSADA, 2003). Dans la grille remplie pour chaque manuel par l'équipe nationale concernée, figurent le nombre de pages du manuel consacrées à l'évolution et à l'évolution humaine en particulier, les titres des chapitres et paragraphes, diverses caractéristiques sur les schémas représentant les origines de l'espèce humaine. Ainsi la place occupée par l'espèce humaine dans le schéma teste l'anthropocentrisme et le finalisme, la présence ou non d'éléments indiquant l'incertitude teste le dogmatisme, et le genre des représentations de l'espèce humaine teste l'androcentrisme. La présence de certains mots-clés comme DARWIN, LAMARCK ou «sélection naturelle» est comptabilisée dans tous les chapitres consacrés à l'évolution du manuel étudié, cela permet de mesurer un des éléments caractéristiques du dogmatisme, la dépersonnalisation souvent associée à la décontextualisation des apports scientifiques (CHEVALLARD, 1985).

\section{Analyse des programmes}

Cette analyse a mis en évidence une très grande disparité d'un pays à l'autre. Plusieurs situations ont été rencontrées :

1) Absence totale de l'évolution, au moment de notre étude, en Algérie et au Maroc.

2) Absence de ce thème dans les programmes en vigueur mais présence dans les manuels scolaires, au Liban. Ce thème avait en effet été introduit après la fin de la guerre civile, mais il a ensuite fait partie des allègements de programme. On le retrouve donc dans les manuels scolaires mais sans qu'il soit enseigné en classe.

3) Traitement réduit à un seul niveau scolaire de Biologie, à la fin du cursus en Tunisie, dans les classes terminales. 
4) Traitement étendu sur plusieurs niveaux scolaires : à Malte et à Chypre sur 3 niveaux, en Italie sur 4 niveaux et en France sur 6 niveaux. enseignée en classe terminale en fin de programme après la génétique, mais elle n'a pas été l'objet de questions dans les épreuves du bac. À partir de 1979, elle fut enseignée en première année du lycée dans la partie géologie dans le chapitre paléontologie sous le titre « Étude de l'évolution d'un groupe animal (au choix du professeur) » et « Esquisse de l'évolution de l'Homme; à partir de documents paléontologiques et archéologiques maghrébins et africains ». À partir de 1994, ce thème a été supprimé des programmes. Au moment du projet Biohead-Citizen, le thème de l'évolution n'était donc plus abordé mais l'évolution biologique a été introduite à nouveau dans les programmes lors de la réforme de 2007 pour l'enseignement secondaire par l'étude d'un exemple d'évolution d'espèces en classe terminale. Cette réintroduction s'appuie sur la Charte de l'éducation et la formation. Elle stipule que le système éducatif du Royaume du Maroc se fonde sur les principes et les valeurs de la foi islamique qui reste ouverte à la science et à la connaissance.

13 L'évolution biologique est enseignée en Tunisie depuis 1991. Au cours de la réforme des programmes tunisiens de juillet 1991, de nombreuses notions et concepts relatifs à l'évolution ont été introduits à partir de la 3e année secondaire (14-15 ans) jusqu'en classe terminale (18-19 ans). Le cas de l'évolution humaine était traité uniquement en classe terminale dans un chapitre propre. À partir de 2002-2003, les contenus ont progressivement été réduits (réforme d'allègement des programmes) et l'hominisation a été l'une des premières parties à être supprimée.

14 C'est en Algérie que peut actuellement être identifié un blocage quant à l'introduction de ce thème dans les programmes. Il était abordé de façon très succincte dans le programme de philosophie de Terminale, mais il en a été supprimé il y a deux ans.

\section{Analyse des chapitres relatifs à l'évolution dans les manuels scolaires}

En Algérie, conformément au programme en vigueur en 2004, aucun manuel de science ne traite de la théorie de l'évolution. Dans le manuel d'histoire qui s'adresse aux élèves de 12 ans, un premier chapitre est consacré à l'Afrique du Nord préhistorique où sont présentés les restes osseux ou lithiques des hommes préhistoriques: seules les approches culturelles et historiques y sont proposées; aucune approche biologique évolutionniste n'y est présente. 
16 À Chypre, deux manuels de science présentent des éléments sur l'évolution. Le premier concerne les élèves de 15 à 16 ans. Il comporte 11 pages sur le sujet avec 3 chapitres, un sur le fait de l'évolution, un sur le mécanisme de l'évolution et un sur l'évolution humaine (3 pages). Des données historiques sont présentées brièvement; LAMARCK, DARWIN et WALLACE sont cités; la sélection naturelle est abordée. Le second manuel de 297 pages, pour les élèves de 16 à 17 ans, présente 22 pages sur l'évolution dont 7 portent sur l'évolution humaine. Le sujet était beaucoup plus développé dans les programmes précédents. Les représentations de la famille humaine n'échappent pas aux stéréotypes : les espèces d'hominidés sont chacune représentées par un individu de sexe masculin et se succèdent linéairement jusqu'à Homo sapiens. Les individus ont la peau qui s'éclaircit lors de « la course » de l'évolution. En plus d'erreurs scientifiques (l'évolution humaine n'est pas une suite linéaire d'espèces), des messages idéologiques sont implicitement identifiables dans ces représentations, tels que le finalisme (l'évolution humaine tend vers l'émergence d'Homo sapiens, qui en apparaît comme la finalité), l'occidentalisme (''image d'Homo sapiens est un homme blanc, de type occidental), et le sexisme (seuls des hommes, jamais des femmes, illustrent ces séries évolutives).

17 En France, les quatre éditions de manuels les plus utilisées en classe terminale ont été analysées. Le thème de l'évolution, en adéquation avec les programmes y est largement couvert: de $20 \%$ à $36 \%$ des pages du manuel dont 13 à 19 pages sont consacrées à l'évolution humaine. L'illustration est très importante avec en moyenne 8 schémas d'évolution. Malgré le traitement important de cette thématique, DARWIN est cité dans un seul manuel sur les quatre, aucun autre scientifique n'est nommé, alors que les mécanismes de l'évolution, dont la sélection naturelle, sont étudiés conformément au programme. Les représentations de l'homme dans les arbres d'évolution n'échappent pas aux stéréotypes. Toutes les représentations proposent un individu occidental de genre masculin comme type d'Homo sapiens. Ces manuels expriment ainsi une double tendance. D'une part s'observe une modernisation importante des contenus scientifiques, avec la quasi-disparition des lignées humaines signalées dans les pays précédents, successions linéaires qui étaient majoritaires chez les mêmes éditeurs jusque vers les années 1990 ; elles sont aujourd'hui remplacées par des schémas phylogénétiques, arbres d'évolution présentant les relations de parenté entre espèces sans indication de temps, ou par des schémas stratigraphiques présentant la répartition des espèces fossiles dans le temps, sans lien entre elles, ou par des schémas mixtes qui associent les approches stratigraphiques et phylogénétiques (QUESSADA et CLÉMENT, 2005). Mais, d'autre part, subsistent des implicites idéologiques puisque, dans les arbres évolutifs modernisés, ce sont très majoritairement des hommes mâles blancs occidentaux qui représentent les humains. Une troisième tendance peut être identifiée, qui différencie les manuels français de leurs homologues italiens: la quasi disparition de toute référence historique ou épistémologique. Alors que LAMARCK et DARWIN, ainsi que leurs apports respectifs, étaient présentés dans les manuels des mêmes éditeurs jusque dans les années 1980, ces références ont disparu. Seuls les contenus scientifiques actuels sont présentés, dépersonnalisés, dogmatisés pour reprendre certaines caractéristiques de la transposition didactique (CHEVALLARD, 1985).

En Italie, cinq manuels proposés pour les élèves de 14 à 18 ans ont également fait l'objet d'une analyse avec la grille élaborée dans le cadre du projet Biohead-Citizen. Le volume du traitement de l'évolution du vivant est assez différent suivant le manuel, de 32 à 112 pages. Par contre, le faible développement de l'évolution humaine se retrouve dans tous 
les manuels, avec uniquement 2 à 4 pages consacrées à ce sujet. En revanche, l'approche historique est approfondie. Outre LAMARCK et DARWIN, LINNÉ, BUFFON, HUTTON, LYELL, CUVIER , AGASSiz, MALThus, WALLACE, GOULD et ELDREDGe sont cités. Cette approche historique s'accompagne d'une véritable étude épistémologique où les différents mécanismes d'évolution et les modèles évolutifs proposés par les scientifiques sont présentés. Cette vigilance épistémologique peut se traduire dans les illustrations originales et est à même de susciter la réflexion. Elle n'est hélas pas toujours conservée quand il s'agit des schémas d'évolution, où nous retrouvons « la lignée humaine » transformiste et finaliste, porteuse des mêmes implicites idéologiques que ceux que nous venons d'indiquer pour les manuels chypriotes. Les manuels italiens semblent donc témoigner d'une culture particulière, à la fois ouverte sur l'épistémologie et l'histoire des sciences, mais ne poussant pas à son terme la vigilance épistémologique (persistance des lignées humaines « classiques »).

19 Au Liban, bien que n'étant plus traité, le thème de l'évolution se retrouve dans les manuels scolaires toujours en usage pour le reste du programme et représente $15 \%$ des pages du manuel avec un tiers consacré à l'évolution humaine. Darwin et la sélection naturelle sont présents, ainsi que de nombreux autres mécanismes d'évolution comme la dérive génique, causée par des phénomènes aléatoires et indépendante des mutations et de la sélection naturelle ou encore l'isolement géographique d'une population, qui peuvent être aussi à l'origine de la création d'une nouvelle espèce. La supériorité de l'homme est affirmée dans les schémas qui le placent au bout du chemin ou encore au sommet. Sa représentation est celle d'un individu de sexe masculin habillé en costume occidental ou nu. Le contenu de ces manuels témoigne donc de la même double tendance que celle identifiée dans les manuels français, dont ils se sont souvent inspirés: la modernisation des connaissances et le maintien d'implicites idéologiques (finalisme, occidentalisme, sexisme). La forte présence de l'évolution humaine témoigne de la volonté de ceux qui ont construit programmes et manuels juste après la guerre civile, de développer au Liban un enseignement proche de celui des pays européens ou américains. Mais l'allègement des programmes, qui a suivi, a montré que c'était encore mal ou non accepté par une partie de la société libanaise. Il sera intéressant d'analyser les contenus, actuellement en préparation, qui introduisent à nouveau ce thème.

À Malte, les deux manuels en usage sont anglais, datent de 1986 et s'adressent aux élèves de 13 à 16 ans. Ils consacrent tous deux seulement $1 \%$ du manuel à l'évolution. Si l'un des deux présente un chapitre "Variation et Sélection" classique sur ce thème, les 5 dernières pages du second manuel (ROBERTS, 1986) sont plus originales dans leur approche. La théorie de l'évolution y est attribuée à Charles DARWIN et à WALLACE. Un paragraphe s'intitule «Creation or evolution? », l'auteur y propose une position qui est à la fois créationniste et évolutionniste : «It is possible that God's way of creating living things may have been through a process of evolution ». Il conclut le paragraphe en précisant que c'est une question très difficile à laquelle nous ne pourrons jamais avoir la réponse. Ce traitement en parallèle des croyances et des théories scientifiques laisse pour le moins perplexe sur la rigueur épistémologique de l'auteur. Le paragraphe suivant s'intitule paradoxalement "Évidence» et présente trois arguments en faveur de la théorie de l'évolution: les fossiles, les caractéristiques communes du vivant et les structures homologues des espèces issues d'un ancêtre commun avec l'exemple du membre des tétrapodes tantôt nageoire chez la baleine, aile chez l'oiseau ou bras chez l'homme. Ce sont les arguments classiques déjà développés au XIXe siècle. Les données génétiques ou moléculaires, développées surtout vers la fin du XXe siècle et qui donnent 
une très forte cohérence à la théorie, ne sont pas utilisées. Une partie de l'explication vient sans doute de la date de publication de ces ouvrages, près de 20 ans avant ceux que nous avons analysés dans les autres pays, à l'aube du grand succès de la biologie moléculaire.

Au Maroc, dans le manuel de la classe terminale de 2007, un ensemble de 47 pages sur 295 au total, sont consacrées à l'étude de la variation et de la génétique des populations. Le nom de DARWIN n'y figure pas. Cette partie du programme est très proche des programmes français de 1994 qui traitaient aussi largement la génétique des populations. Dans les deux derniers chapitres, contrairement au choix français de 1994 qui abordait l'évolution humaine, c'est l'évolution du cheval qui est traitée. Elle repose sur l'étude de quelques données paléontologiques ainsi que sur des phénomènes caractéristiques de l'évolution des équidés. L'évolution humaine n'est pas du tout abordée. Il est intéressant de relier cette partie à un programme en vigueur en France en 1958 (23 Octobre 1958 BOEN n $\left.{ }^{\circ} 38\right)$ où un seul aspect de l'évolution des êtres vivants était présent avec l'étude d'un fait paléontologique de l'évolution, la lignée des équidés. Ce nouveau contenu des manuels marocains témoigne donc à la fois de la forte volonté d'enseigner de façon moderne les sciences, mais avec des limites conduisant à réduire la part de l'évolution humaine tout en développant des exemples classiques moins polémiques, comme l'évolution des équidés.

En Tunisie, deux chapitres sont consacrés à l'évolution biologique dans les manuels de science destinés aux élèves de classe terminale de toutes les sections du lycée. Le premier porte sur «quelques faits de l'évolution des êtres vivants » avec l'étude des fossiles, des organes et des molécules homologues. Le second chapitre s'intitule "Mécanisme de l'évolution des êtres vivants» avec «Modifications génétiques et transformation héréditaire de l'espèce » et "la sélection naturelle » qui présente une photographie de Charles DARWIN. La première page du manuel montre deux squelettes, l'un de cheval et l'autre de l'homme, la légende indique "Seraient-ils de lointains cousins? Auraient-ils un ancêtre commun?». Dans la seconde page, deux schémas sont mis en parallèle: un schéma représentant l'hypothèse fixiste et un schéma représentant l'évolution à partir d'un ancêtre commun. Le texte qui l'accompagne distingue les théories de l'évolution du dogme créationniste. Ces contenus, ainsi que leur modification durant ces vingt dernières années, témoignent du même paradoxe que celui signalé ci-dessus pour le Liban et le Maroc: une volonté de développer un enseignement scientifique moderne mais qui se heurte à des résistances à l'enseignement de l'évolution humaine.

\section{Discussion sur les contenus des programmes et des manuels scolaires en lien avec les conceptions des enseignants des huit pays méditerranéens}

Les résultats qui précèdent montrent donc une situation extrêmement contrastée d'un pays à un autre, parmi les huit pays méditerranéens de notre échantillon. Elle peut être mise en lien avec les conceptions des enseignants biologistes de ces huit pays sur le thème de l'évolution. Ces conceptions ont été étudiées dans le cadre du projet Biohead-Citizen par un questionnaire qui comportait quinze questions relatives à l'évolution (CLÉMENT et QUESSADA, 2008, 2009). 

vie: «Avec laquelle de ces quatre propositions êtes-vous le plus en accord? 1) Il est certain que l'origine de la vie est le résultat de phénomènes naturels. 2) L'origine de la vie peut être expliquée par des phénomènes naturels sans avoir besoin de l'hypothèse que Dieu a créé la vie. 3) L'origine de la vie peut être expliquée par des phénomènes naturels qui sont sous le contrôle de Dieu. 4) Il est certain que Dieu a créé la vie ». La proposition 1 correspond à une conception évolutionniste dogmatique, la proposition 2 à une conception évolutionniste non dogmatique, la proposition 3 témoigne d'une conception à la fois évolutionniste et créationniste, tandis que la proposition 4 correspond à une position créationniste radicale. marocains, $70 \%$ des enseignants biologistes libanais et tunisiens ont choisi la proposition 4 correspondant à une conception créationniste radicale. Ces pourcentages sont très proches pour une question relative cette fois à l'origine de l'espèce humaine. Dans ces quatre pays, presque tous ces enseignants croient en Dieu : sur une échelle de 1 « je ne crois pas » à 5 « je crois », de 95\% (en Tunisie) à 99\% (en Algérie) cochent la case 5. Leur degré de pratique religieuse est un peu plus faible, et plus variable d'un pays à un autre: $49 \%$ en Tunisie, $77 \%$ au Liban, $82 \%$ au Maroc et $88 \%$ en Algérie. Cette variation correspond assez bien avec l'importance de l'enseignement de l'évolution dans ces quatre pays: stabilisée en Terminale en Tunisie, en cours de réintroduction en Terminale au Liban et au Maroc, et toujours totalement absente en Algérie. Nos résultats suggèrent donc que plus la pratique religieuse est importante dans un pays (du moins chez les enseignants, ce qui peut être considéré comme un indicateur fiable de la situation dans le pays), plus le thème de l'évolution a des difficultés à apparaître dans les programmes et manuels scolaires. Il semble que les difficultés à introduire le thème de l'évolution dans les programmes et manuels scolaires soient plus liées à l'emprise de la religion qu'à la religion elle-même (CLÉMENT et QUESSADA 2008, 2009) : au Liban, les enseignants chrétiens ont des conceptions statistiquement non différentes de celles des enseignants musulmans. Et au Gabon (résultats plus récents non encore publiés), les 300 enseignants interrogés sont chrétiens mais tout aussi créationnistes radicaux que leurs collègues algériens de religion musulmane.

Parmi les quatre pays européens, la France occupe une place à part : moins de $2 \%$ des 750 enseignants interrogés sont créationnistes radicaux, alors qu'ils sont $15 \%$ en Italie et $25 \%$ à Chypre ou à Malte. Ces résultats sont en lien avec la proportion d'enseignants agnostiques ou athées dans ces échantillons d'enseignants (51\% en France, $12 \%$ en Italie, $4 \%$ à Chypre et $0,5 \%$ à Malte) et sont inversement proportionnels à la proportion d'enseignants pratiquants (6\% en France, 29\% en Italie et à Chypre, 54\% à Malte). Ces corrélations sont une clé intéressante pour interpréter les différences observées dans les manuels scolaires d'un pays à un autre dans notre échantillon de huit pays méditerranéens. Ainsi en France, où l'emprise de la religion est la moins forte, et où plus de $98 \%$ des enseignants sont évolutionnistes, l'évolution est abondamment et scientifiquement enseignée, avec même une tendance à la dogmatisation, excluant l'approche épistémologique et historique. A l'autre extrême de ces quatre pays européens et méditerranéens, Malte est celui où les enseignants sont les plus croyants ( $84 \%$ cochent la case 5), les plus pratiquants (54\%) et les plus créationnistes : $82 \%$ cochent les cases 3 ou 4 en répondant à la question sur l'origine de la vie, mais $57 \%$ cochent la case 3 qui est à la fois créationniste et évolutionniste, Dieu contrôlant les processus de l'évolution analysés 
par les biologistes et enseignés à l'école. Or c'est à Malte que nous avons rencontré le seul manuel scolaire exprimant la possibilité de cette position à la fois créationniste et évolutionniste.

27 Au total, la comparaison que nous avons tentée entre huit pays méditerranéens a donc surtout opposé les pays du Nord et du Sud de la Méditerranée. On note une relation entre le traitement de l'évolution et notamment l'évolution humaine dans l'enseignement, et l'ancrage des pratiques religieuses dans le pays. L'évolution est beaucoup plus acceptée et enseignée au Nord qu'au Sud, et, même au Nord, elle est enseignée de façon moins complète, moins actualisée, en intégrant moins les origines de l'espèce humaine, dans les pays où la religion a le plus d'emprise. Ces résultats et interprétations nous poussent à suggérer que l'enseignement de l'évolution n'élude pas ce qui en constitue une condition structurante : la clarification des différences entre ce qui relève de la science et ce qui relève de la religion. Clarification qui permet d'accepter qu'on puisse être, à l'instar de DOBZHANSKY (1973) auteur du célèbre article, titré «Nothing in biology makes sense except in the light of evolution » à la fois croyant et totalement évolutionniste (CLÉMENT et al., 2009). Une approche historique des difficultés de l'acceptation des théories de l'évolution serait une bonne opportunité pour aborder cette clarification dans les manuels scolaires.

Cependant, notre vigilance épistémologique ne peut se limiter à cette suggestion. En effet, les analyses de manuels scolaires que nous avons présentées ne font pas que différencier les huit pays méditerranéens choisis. Elles soulignent des points de convergence dans la survivance de certains implicites idéologiques. Ainsi la conception linéaire et finaliste de l'évolution humaine qui prévalait jusqu'au moins le milieu du XXe siècle, se retrouve dans de nombreux manuels, malgré l'avancée des recherches qui s'accordent aujourd'hui sur une conception buissonnante et contingente de l'évolution des hominidés, conception dégagée du finalisme, où l'espèce humaine actuelle, dernier représentant des hominidés, figure sur l'un des nombreux rameaux dans l'arbre d'évolution de la famille humaine. Et ces schémas de lignée humaine sont empreints de sexisme et d'occidentalisme, ne figurant que des hommes blancs. Ces survivances se font certainement à l'insu des auteurs et des éditeurs des manuels, elles tiennent en partie aux pratiques d'édition qui reprennent des éléments des éditions précédentes, notamment iconographiques pour les conserver à l'identique ou les modifier légèrement. Mais elles tiennent aussi en partie à la persistance d'idéologies dans ce type d'images familières. C'est ici une vigilance citoyenne qu'il s'agit de développer, portant sur les valeurs et pratiques sociales associées à des connaissances scientifiques. Nous l'avons mise en œuvre dans le projet Biohead-Citizen (2004-2008 : voir par exemple QUESSADA et al. 2008 sur le thème de l'évolution), le même type de vigilance ayant aussi été développé par d'autres auteurs, par exemple l'analyse critique de manuels scolaires en Afrique (BRUGEILLES et CROMER 2005, 2009).

Soulignons enfin que, dans les manuels scolaires, le traitement des contenus scientifiques oscille entre deux pôles, le pôle dogmatique et le pôle non dogmatique. Cela est très net en ce qui concerne la contextualisation de la théorie de l'évolution: DARWIN est presque totalement ignoré des manuels français et marocains. Il est présent dans les manuels des six autres pays qui traitent de l'évolution, particulièrement en Italie où l'histoire des concepts relatifs à l'évolution est très détaillée. Lors de la transposition didactique, le manque de perspective historique, l'absence de référence à des scientifiques allant jusqu'à une dépersonnalisation totale contribue a contrario à dogmatiser le discours, à présenter une science figée. L'enseignement ignore alors que la science est le produit 
d'une activité humaine avec les contraintes et les obstacles propres à toutes activités humaines, et tout au contraire il viserait à dévoiler une "vérité préexistante" universelle. Du coup, l'objet même de l'enseignement scientifique, avec problématisation et rectifications du modèle explicatif en vigueur, est perdu. C'est au contraire en contextualisant l'avancée des connaissances dans ce domaine qu'on peut mieux aider les élèves à différencier ce qui tient de la science et ce qui tient de la religion, du mythe et des croyances.

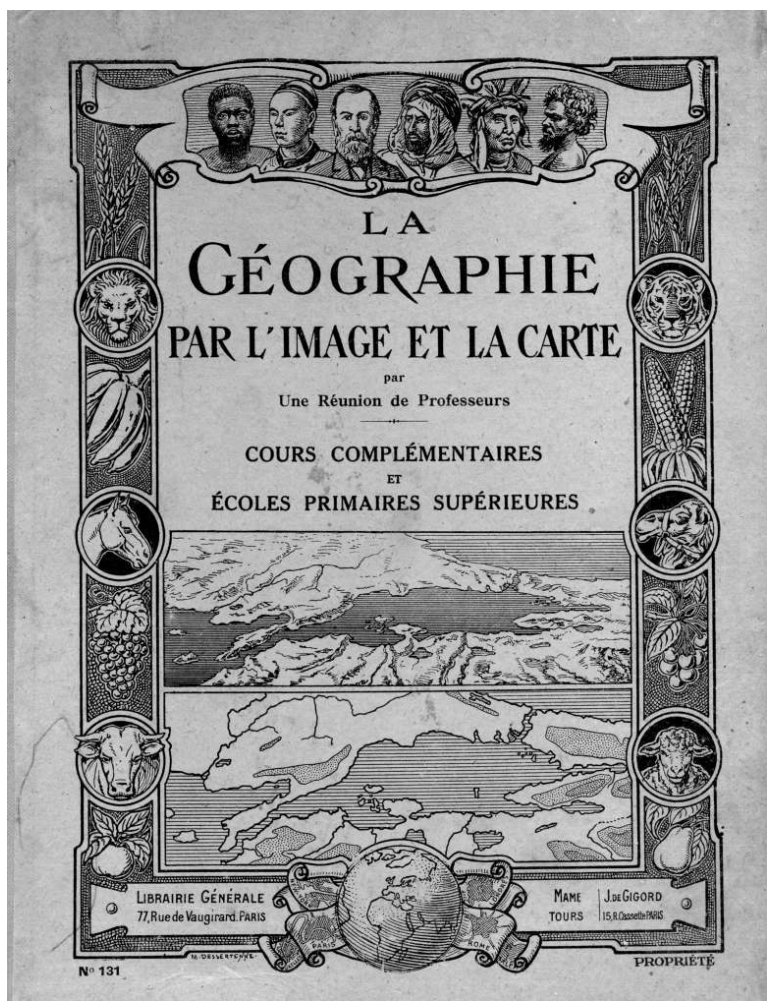

La géographie par l'image et la carte, cours complémentaire et écoles primaires supérieures, par Une réunion de professeurs. Paris : Librairie générale, 1933, p. 256 - Couverture. Source : CEDRHE.

\section{BIBLIOGRAPHIE}

BRUGEILLES C. et CROMER S., Analyser les représentations du masculin et du féminin dans les manuels scolaires. Paris : CEPED, 2005

BRUGEILLES C. et CROMER S., Promoting gender equity through textbooks. Paris : UNESCO (in English or in French), 2009

CHEVALLARD Y., La transposition didactique. Grenoble : La Pensée sauvage, 1985

CHOPPIN A., Les manuels scolaires : histoire et actualité. Paris : HACHETTE Éducation, 1992 
CLEMENT P., Science et idéologie : exemples en didactique et épistémologie de la biologie. Actes du Colloque Sciences, médias et société. ENS-LSH, 2004, p.53-69

http://sciences-medias.ens-lsh.fr

CLEMENT P., DRAMISINO J.P. et ESTEVES F., Creationist conceptions in 14 countries. How to teach evolution to french students coming from immigration? Communication au Colloque BioEd 2009, Evolution in action, Christchurch (New Zealand), 2009

CLEMENT P., et QUESSADA M.P., Les convictions créationnistes et/ou évolutionnistes d'enseignants de biologie : une étude comparative dans 19 pays. Nature, sciences and sociétés, 16, 2008, p. 154-158

CLEMENT P., et QUESSADA M.P., Creationist beliefs in europe. Science, 324, $26: 1644,2009$

DOBZHANSKY T., Nothing in biology makes sense except in light of evolution. American biology teacher, 2009, p. 35,125-129

HARFOUCH Z., Résultats de l'analyse des manuels scolaires libanais et des conceptions des enseignants à propos de l'origine de l'Homme. Recherches pédagogiques (Faculté de pédagogie Université libanaise) numéro spécial, 2008, p. 31-49

IAP InterAcademy panel, IAP Statement on the teaching of evolution, 2006

http://www.interacademies.net/Object.File/Master/6/150/Evolution\%20statement.pdf

MATHY P., Les théories de l'évolution dans les manuels de biologie. In MATHY P. (Ed.), Donner du sens au cours de sciences. Des outils pour la formation éthique et épistémologique des enseignants, 1997, pp. 176-231. Bruxelles : DE BOECK Université.

PICQ P., Une évolution buissonnante. Pour la Science, 300, 2002, p. 32-36

QUESSADA M.P., Les origines de l'homme : approche épistémologique et étude de la transposition didactique dans l'enseignement des sciences naturelles aux XIXe et XXe siècles. DEA Construction des savoirs scientifiques, Université Montpellier II, Université Claude BERNARD Lyon I, École doctorale : informations et informatique pour la société, 2003

QUESSADA M.P., L'enseignement des origines d'Homo sapiens, hier et aujourd'hui, en France et ailleurs : programmes, manuels scolaires, conceptions des enseignants. Université de Montpellier II, 2008, 430 pages.

http://tel.archives-ouvertes.fr/tel-00353971/fr/

QUESSADA M.P. et CLEMENT P., Introduction du concept d'évolution humaine buissonnante dans les manuels scolaires de Sciences de la vie et de la terre de Terminale scientifique. In Actes 4es Rencontres de l'ARDIST. Lyon, INRP, 2005, p. 293-300

QUESSADA M.P., et CLEMENT P., An epistemological approach to french curricula on human origin during the XIXth et XXth centuries. Science et Education, 16, 9-10, 2007, p. 991-1006

QUeSSADA M.P., et CLEMENT P., OERKE, B. et VAlente, A., Human evolution in science textbooks: A survey in eighteen. Science education international, 19, (2), 2008, p. 147-162

SKоOG G., The coverage of human evolution in high school biology textbooks in the XXth Century and in current state science standards. Science and education, 14, 2005, p. 395-422

TIDON R. et LEWONTIN C., Teaching evolutionary biology. Genetics and molecular biology, 27, 2004, p. $1-8$ 


\section{NOTES}

1. Projet de recherche financé par la Communauté Européenne (FP6, Priority 7) en 2004-2008, coordonné par G. Carvalho, P. Clément \& F. Bogner.

\section{RÉSUMÉS}

Les manuels scolaires de biologie de huit pays méditerranéens abordent le thème de l'évolution de façon très différente. En Algérie et au Liban, il n'est pas ou plus traité. En Tunisie, le thème est traité en Terminale, mais sans chapitre spécifique à l'évolution humaine. Au Maroc, l'évolution vient d'être réintroduite en Terminale, sans l'évolution humaine. Le thème est plus largement couvert à Chypre et à Malte, et surtout en Italie et en France. Quand il est abordé, on y retrouve des valeurs implicites telles que l'anthropocentrisme, le finalisme, l'occidentalisme, le dogmatisme.

The topic Evolution strongly differs among the school textbooks of biology in eight Mediterranean countries. In Algeria and Lebanon, it is not (or no more) present. In Morocco also, but it is just now reintroduced (nevertheless without the human origin). Poorly taught in Tunisia, the topic is more present in Cyprus and Malta, and even more in Italian and French textbooks. When it is present, we identified some implicit values as anthropocentrism, goal-ended evolution, occidentalism and dogmatism.

\section{INDEX}

Mots-clés : biologie, créationnisme, enseignant, évolution, manuel scolaire Keywords : biology, creationism, evolution, teacher, textbook

\section{AUTEURS}

\section{MARIE-PIERRE QUESSADA}

LIRDEF, IUFM, Université Montpellier 2, France

\section{PIERRE CLÉMENT}

Université Lyon 1, France

SABAH SELMAOUI

EREF, École Normale Supérieure, Université Cadi Ayyad, Marrakech, Maroc

\section{ADRIANA VALENTE}

Institut de recherche sur la population et les politiques sociales, CNR, Rome, Italie 